\title{
Clinical Characteristics and Prognosis of Fetal Left Ventricular Noncompaction in Japan
}

\author{
Sayaka W Ozawa, MD, PhD; Shinya Takarada, MD; Mako Okabe, MD, PhD; \\ Nariaki Miyao, MD, PhD; Hideyuki Nakaoka, MD, PhD; Keijiro Ibuki, MD, PhD; \\ Fukiko Ichida, MD, PhD; Keiichi Hirono, MD, PhD; Fetal CM Study Collaborators
}

\begin{abstract}
Background: Left ventricular noncompaction (LVNC) is morphologically characterized by numerous prominent trabeculations and a severely thickened, two-layered myocardium. The fetal onset of LVNC has rarely been described.

Methods and Results: We conducted nationwide retrospective surveys on fetal cardiomyopathy (CM) in Japan from 2010 to 2016 , from which 38 fetal patients with CM were enrolled, including 16 patients with LVNC. The rate of diagnostic concordance was $56.3 \%$ between fetal and postnatal visits in LVNC patients. The increase in the ratio of noncompacted to compacted (N/C) myocardium was time-dependent throughout the fetal period till birth (LV lateral: $1.6 \pm 0.1$ to $2.8 \pm 0.2$; LV apex: $2.0 \pm 0.1$ to $3.2 \pm 0.2$ ). Of all fetuses, 16 $(42.1 \%)$ died or underwent heart transplantation (HT), with 3 intrauterine deaths. Lower fetal cardiovascular profile score (odds ratio, 26.9; $\mathrm{P}=0.0266$ ) was a risk factor for death or $\mathrm{HT}$. $\mathrm{N} / \mathrm{C}$ ratio $\geq 1.6$ at the apex at the first visit was a significant predictor of $\mathrm{LVNC}$ (odds ratio, 47.8; $P=0.0113$ ).
\end{abstract}

Conclusions: This is the first study to reveal the etiology of fetal CM based on results from a nationwide survey in Japan, highlighting the difficulty of diagnosing LVNC in fetal patients. To better understand and manage fetal CM, novel diagnostic criteria of LVNC in fetus should be established.

Key Words: Cardiovascular profile score; Fetal echocardiography; Left ventricular noncompaction; N/C ratio

$\mathbf{L}$ eft ventricular noncompaction (LVNC) is morphologically characterized by a severely thickened, twolayered myocardium and numerous prominent trabeculations and deep intertrabecular recesses that communicate with the LV cavity. ${ }^{1}$ The clinical features of LVNC are highly variable: some patients experience no symptoms, whereas others might experience progressive deterioration in cardiac function that results in congestive heart failure, thromboembolism, arrhythmias, and sudden cardiac death. ${ }^{1-4}$ Therefore, early diagnosis and appropriate management are crucial.

The ability to detect LVNC in the fetus has been rarely described in this population. Although distinct criteria have been established for diagnosing LVNC after birth, those for fetal LVNC diagnosis remain unidentified. ${ }^{5} \mathrm{~A}$ previous study reported that continual morbidity and mortality rates occur in fetal cases of LVNC, even if treated with appropriate care perinatally and during childhood. ${ }^{6,7}$ Currently, there are no published data available on the long-term outcomes of fetuses diagnosed with LVNC.

\begin{abstract}
Editorial p 106
We conducted nationwide retrospective surveys of fetuses with cardiomyopathies (CMs) in Japan January 2010 to December 2016, by which we aimed to elucidate the clinical features, diagnosis, and prognosis of fetal LVNC.
\end{abstract}

Methods
Subjects
We conducted a multicenter, retrospective, observational
study by inviting 131 hospitals with a fetal or pediatric
cardiology department in Japan to participate in a survey
of fetal patients with CMs from. After agreeing to partici-
pate, we sent a detailed questionnaire and DVD to 20
hospitals to obtain fetal and postnatal clinical and echo-
cardiographic data. The questionnaire elicited maternal,
fetal, and postnatal information, such as maternal and

Received November 6, 2020; revised manuscript received May 30, 2021; accepted June 15, 2021; J-STAGE Advance Publication released online August 3, 2021 Time for primary review: 16 days

Department of Pediatrics, Graduate school of Medicine, University of Toyama, Toyama (S.W.O., S.T., M.O., N.M., H.N., K.I., K.H.); Department of Pediatrics, International University of Health and Welfare, Tokyo (F.I.), Japan

The Fetal CM Study Collaborators are listed in Supplementary File 1.

Mailing address: Keiichi Hirono, MD, PhD, Department of Pediatrics, Graduate School of Medicine, University of Toyama, 2630 Sugitani, Toyama 930-0194, Japan. E-mail: khirono1973@gmail.com

All rights are reserved to the Japanese Circulation Society. For permissions, please e-mail: cj@j-circ.or.jp

ISSN-1346-9843 


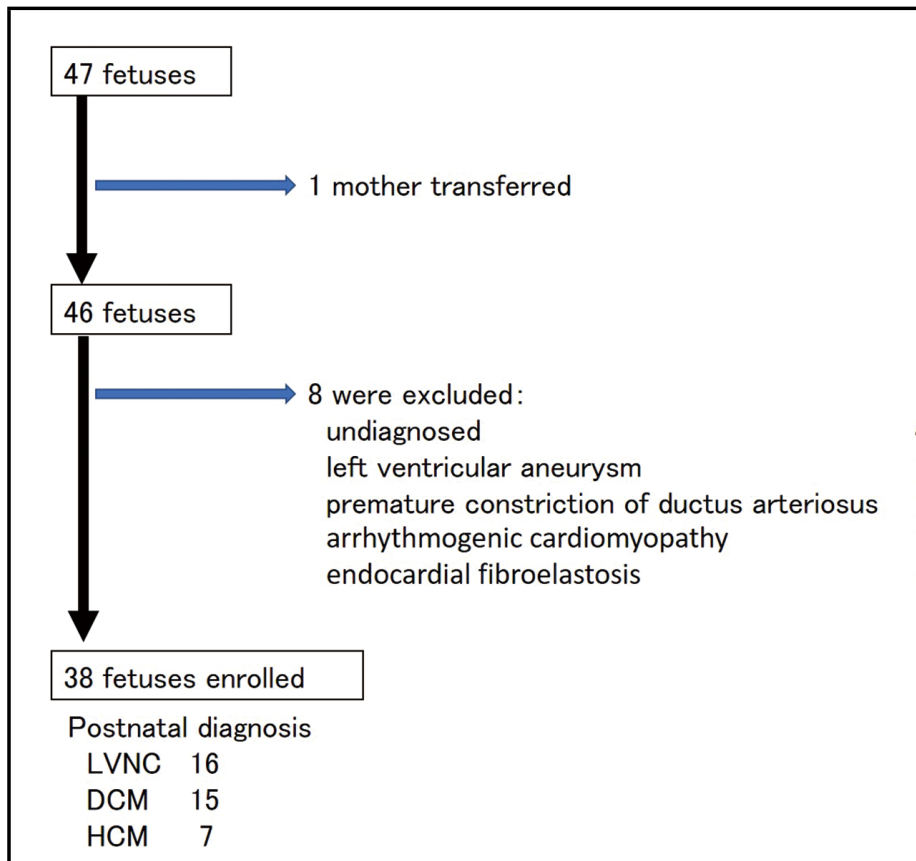

Figure 1. Flow chart of the enrolled fetuses. DCM, dilated cardiomyopathy; HCM, hypertrophic cardiomyopathy; LVNC, left ventricular noncompaction.

family histories; diagnosis and prognosis; echocardiographic data in the fetal and postnatal periods; treatment after birth. The study protocol conformed to the ethical guidelines of the Declaration of Helsinki 1975 and was approved by the Research Ethics Committee of the University of Toyama (approval no. R2016020), Toyama, Japan.

\section{Measurements and Definitions}

The LVNC diagnosis was based on the characteristic twolayered myocardium with a noncompacted to compacted $(\mathrm{N} / \mathrm{C})$ ratio $>2.0$ at end-diastole, and the observed disease process in $>1$ ventricular wall segment. ${ }^{7,8}$ Dilated cardiomyopathy (DCM) is characterized by cardiac dysfunction and ventricular enlargement. For a fetus, cardiac dysfunction was defined as left ventricular fraction shortening (LVFS) $<28 \%$ and ventricular enlargement was defined as cardiothoracic area ratio (CTAR) $\geq 35 \%$ unrelated to structural heart disease. Cardiomyopathy cases associated with positive anti-SS-A AV block and maternal diabetes were excluded. Hypertrophic cardiomyopathy (HCM) is clinically defined as obvious ventricular hypertrophy and a diastolic ventricular wall thickness $>2.0 \mathrm{z}$-scores. The z-scores of the interventricular septum (IVS) and posterior wall (PW) were measured at end-diastole. ${ }^{9}$ We defined all the described cardiomyopathies in the prenatal and postnatal periods, and enrolled the patients who satisfied this definition. Hydrops fetalis is a condition characterized by abnormal accumulation of fluids in $\geq 2$ compartments of the fetus such as skin edema, pleural effusion, and pericardial effusion. We used the cardiovascular profile score (CVPS) to assess the severity of fetal heart failure. ${ }^{10}$ The score includes 5 echocardiographic variables, and a score of 10 points indicates normal cardiac function (Supplementary Table 1). Two independent reviewers (S.W.O., K.H.), blinded to each other's measurements, analyzed the echocardiographic data. Intra- and interobserver variability were evaluated by repeating the analysis 8 weeks later by the same observer and a second investigator.

\section{Endpoints}

The primary endpoints were death or heart transplantation (HT), and the secondary endpoint was prenatal diagnosis of LVNC.

\section{Statistical Analysis}

Distributed data are summarized as means and standard deviations, whereas nonparametric data are presented as medians and interquartile range. $\chi^{2}$ or Fisher's exact test was used for comparing the distributions of variables according to CM type, Tukey-Kramer method for numeric variables, and paired t-test for overtime variables. Cox's proportional-hazards model was used to assess risk factors for death. All the variables were first compared using separate univariate models, then a multivariate model. Receiver operating characteristic (ROC) curve analysis was conducted to determine the optimal cutoff levels of the number of derivations obtained from demographic and echocardiographic data to predict LVNC. Survival curves were plotted using Kaplan-Meier survival estimates; distributions of time to event outcomes according to CM type were compared using the log-rank test. We defined as zero month from the date of fertilization at event-free survival curve. Statistical significance was set at $\mathrm{P}<0.05$. Statistical calculations were performed using JMP Pro 14.2.0.

\section{Results}

\section{Clinical Features}

We collected data of 47 fetuses with CM. We excluded 9 fetuses from the study because of other than CM, arrhythmogenic cardiomyopathy, and endocardial fibroelastosis (Figure 1). Accordingly, 38 fetuses were enrolled in the study: 16 fetuses with LVNC, 15 with DCM, and 7 with HCM. They were diagnosed in the postnatal period. The demographic characteristics of the patients at first visit and outcomes are listed in Table 1 and Supplementary Table 2. Follow-up ranged from 0 days to 64 months (median, 10.5 
Table 1. Baseline Clinical and Echocardiographic Characteristics of the Fetuses With Cardiomyopathy

\begin{tabular}{|c|c|c|c|c|c|}
\hline & $\begin{array}{c}\text { Total } \\
(n=38)\end{array}$ & $\begin{array}{l}\text { LVNC } \\
(n=16)\end{array}$ & $\begin{array}{c}\mathrm{DCM} \\
(n=15)\end{array}$ & $\begin{array}{l}\mathrm{HCM} \\
(\mathrm{n}=7)\end{array}$ & $P$ value \\
\hline Maternal age at diagnosis (years) & $32.0(22-41)$ & $33.5(27-41)$ & $30.5(22-40)$ & $28.0(26-37)$ & 0.091 \\
\hline GA at diagnosis (weeks) & $30(18-39)$ & $29(24-36)$ & $28(22-38)$ & $34(18-39)$ & 0.569 \\
\hline Sex (male) & $22(57.8 \%)$ & $8(50.0 \%)$ & $10(83.3 \%)$ & $4(80.0 \%)$ & 0.148 \\
\hline \multicolumn{6}{|l|}{ Diagnosis opportunity } \\
\hline Fetal heart screening & $22(57.8 \%)$ & $10(62.5 \%)$ & $8(53.3 \%)$ & $4(57.1 \%)$ & 0.874 \\
\hline Hydrops fetalis & $7(18.4 \%)$ & $2(12.5 \%)$ & $4(26.6 \%)$ & $1(14.2 \%)$ & 0.767 \\
\hline Family history of $\mathrm{CM}$ & $10(26.3 \%)$ & $8(50.0 \%)$ & $2(13.3 \%)$ & $0(0 \%)$ & 0.020 \\
\hline Fetal complications & $16(42.1 \%)$ & $6(37.5 \%)$ & $7(46.6 \%)$ & $3(42.8 \%)$ & 0.909 \\
\hline Hydrops fetalis & $17(44.7 \%)$ & $4(25.0 \%)$ & $8(53.3 \%)$ & $5(71.4 \%)$ & 0.082 \\
\hline Congenital heart disease & $12(31.5 \%)$ & 9 (56.3\%) & $2(13.3 \%)$ & $1(14.3 \%)$ & 0.024 \\
\hline Arrhythmia & $4(10.5 \%)$ & $3(18.5 \%)$ & $0(0 \%)$ & $1(14.2 \%)$ & 0.218 \\
\hline \multicolumn{6}{|l|}{ Echocardiography } \\
\hline Heart rate (beats/min) & $140.4 \pm 10.6$ & $141.1 \pm 9.7$ & $136.6 \pm 10.8$ & $150.0 \pm 8.8$ & 0.168 \\
\hline CTAR (\%) & $39.5 \pm 7.3$ & $40.1 \pm 6.0$ & $39.2 \pm 6.5$ & $39.0 \pm 11.9$ & 0.927 \\
\hline CVPS & $7.3 \pm 1.7$ & $8.0 \pm 1.6$ & $6.7 \pm 1.7$ & $7.0 \pm 1.0$ & 0.207 \\
\hline Mitral regurgitation & $16(42.1 \%)$ & $2(12.5 \%)$ & $12(85.7 \%)$ & $2(33.3 \%)$ & 0.0001 \\
\hline Tricuspid regurgitation & $22(57.8 \%)$ & $9(56.3 \%)$ & $10(71.4 \%)$ & $3(50.0 \%)$ & 0.660 \\
\hline LVFS (\%) & $22.1 \pm 9.2$ & $24.8 \pm 11.5$ & $18.2 \pm 5.6$ & $26.5 \pm 9.1$ & 0.339 \\
\hline Concordance between pre- and postnatal diagnoses & $23(60.5 \%)$ & $9(56.3 \%)$ & $11(73.3 \%)$ & $3(42.9 \%)$ & 0.386 \\
\hline Death/HT & $16(42.1 \%)$ & $4(25.0 \%)$ & $11(73.3 \%)$ & $1(14.3 \%)$ & 0.004 \\
\hline Intrauterine death & $3(7.9 \%)$ & $1(6.3 \%)$ & $2(13.3 \%)$ & $0(0 \%)$ & 0.525 \\
\hline
\end{tabular}

Values are $\mathrm{n}(\%)$, mean $\pm \mathrm{SD}$, or median (interquartile range). CM, cardiomyopathy; CTAR, cardiothoracic area ratio; CVPS, cardiovascular profile score; DCM, dilated cardiomyopathy; GA, gestational age; HCM, hypertrophic cardiomyopathy; LVFS, left ventricular fraction shortening; LVNC, left ventricular noncompaction.

\begin{tabular}{|c|c|c|c|c|c|}
\hline & $\begin{array}{c}\text { Total } \\
(n=35)\end{array}$ & $\begin{array}{l}\text { LVNC } \\
(n=15)\end{array}$ & $\begin{array}{c}\text { DCM } \\
(n=13)\end{array}$ & $\begin{array}{l}\mathrm{HCM} \\
(\mathrm{n}=7)\end{array}$ & $P$ value \\
\hline Transvaginal delivery & $16(42.1 \%)$ & $7(46.7 \%)$ & $6(46.2 \%)$ & $3(50.0 \%)$ & 1.000 \\
\hline GA at birth (weeks) & $37(30-40)$ & $37(30-40)$ & $36(32-40)$ & 37 (34-39) & 0.739 \\
\hline Birth weight (g) & $2,808 \pm 543$ & $2,782 \pm 584$ & $2,694 \pm 435$ & $3,102 \pm 616$ & 0.323 \\
\hline Birth height $(\mathrm{cm})$ & $47.2 \pm 2.9$ & $47.1 \pm 3.0$ & $42.2 \pm 2.5$ & $49.7 \pm 2.4$ & 0.932 \\
\hline Apgar score (1 min) & $6 \pm 2.7$ & $6.9 \pm 1.6$ & $5.5 \pm 3.5$ & $4.6 \pm 2.5$ & 0.135 \\
\hline Apgar score $(5 \mathrm{~min})$ & $7.2 \pm 2.5$ & $7.8 \pm 1.9$ & $6.6 \pm 3.4$ & $7.1 \pm 0.7$ & 0.474 \\
\hline Extracardiac involvement & $5(14.2 \%)$ & $1(6.6 \%)$ & $1(7.6 \%)$ & $3(42.8 \%)$ & 0.181 \\
\hline Arrhythmia & $6(17.1 \%)$ & $4(26.6 \%)$ & $1(7.0 \%)$ & $1(14.0 \%)$ & 0.824 \\
\hline \multicolumn{6}{|l|}{ Chest X-ray } \\
\hline CTR & $0.62 \pm 0.07$ & $0.62 \pm 0.06$ & $0.64 \pm 0.1$ & $0.62 \pm 0.06$ & 0.710 \\
\hline Pulmonary congestion & $12(34.2 \%)$ & $4(28.6 \%)$ & $4(44.4 \%)$ & $4(80.0 \%)$ & 0.156 \\
\hline \multicolumn{6}{|l|}{ Echocardiography } \\
\hline LVFS (\%) & $23.5 \pm 14.4$ & $23.7 \pm 9.5$ & $13.1 \pm 8.9$ & $43.4 \pm 17.8$ & 0.027 \\
\hline MR (>moderate) & $8(22.8 \%)$ & $1(16.6 \%)$ & $6(66.6 \%)$ & $1(16.6 \%)$ & 0.005 \\
\hline IVS z-score & $0.23 \pm 1.7$ & $-0.1 \pm 0.4$ & $-0.5 \pm 0.6$ & $2.4 \pm 0.7$ & 0.012 \\
\hline LVPW z-score & $1.9 \pm 2.0$ & $1.5 \pm 0.5$ & $1.9 \pm 0.8$ & $3.0 \pm 1.0$ & 0.432 \\
\hline Treatment (subjects, \%) & $24(26,92.3 \%)$ & $12(13,92.3 \%)$ & $7(8,87.5 \%)$ & $5(5,100 \%)$ & 0.565 \\
\hline Diuretics & $21(87.5 \%)$ & $12(100 \%)$ & $6(85.7 \%)$ & $3(60.0 \%)$ & 0.275 \\
\hline Catecholamine & $20(83.3 \%)$ & $11(91.6 \%)$ & $6(85.7 \%)$ & $3(60.0 \%)$ & 0.457 \\
\hline Ventilator support & $18(75.0 \%)$ & $9(75.0 \%)$ & $5(71.4 \%)$ & $4(80.0 \%)$ & 0.582 \\
\hline$\beta$-blocker & $18(75.0 \%)$ & $10(83.3 \%)$ & $4(57.1 \%)$ & $4(80.0 \%)$ & 0.383 \\
\hline PDE3 inhibitor & $15(62.5 \%)$ & $9(75.0 \%)$ & $5(71.4 \%)$ & $1(20.0 \%)$ & 0.197 \\
\hline ACE inhibitor/ARB & $14(58.3 \%)$ & $9(75.0 \%)$ & $4(57.1 \%)$ & $1(20.0 \%)$ & 0.196 \\
\hline Antiplatelet/anticoagulant drug & $8(33.3 \%)$ & $6(50.0 \%)$ & $1(14.2 \%)$ & $1(20.0 \%)$ & 0.355 \\
\hline PMI & $2(0.08 \%)$ & $2(16.6 \%)$ & $0(0 \%)$ & $0(0 \%)$ & 0.601 \\
\hline
\end{tabular}

Values are $\mathrm{n}(\%)$, mean $\pm \mathrm{SD}$, or median (interquartile range). ACE, angiotensin-converting enzyme; ARB, angiotensin receptor blockers; CTR cardiothoracic ratio; HT, heart transplantation; IVS, interventricular septum; LVPW, left ventricular posterior wall; MR, mitral regurgitation; PDE, phosphodiesterase; PMI, pacemaker implantation. Other abbreviations as in Table 1. 


\section{A}

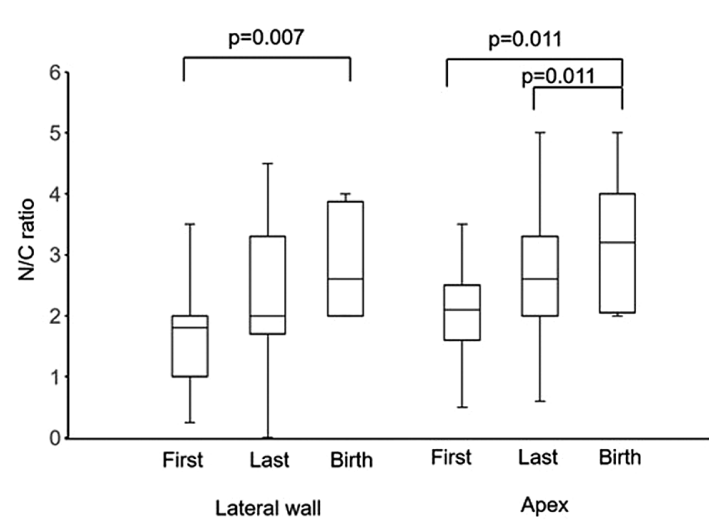

C
B
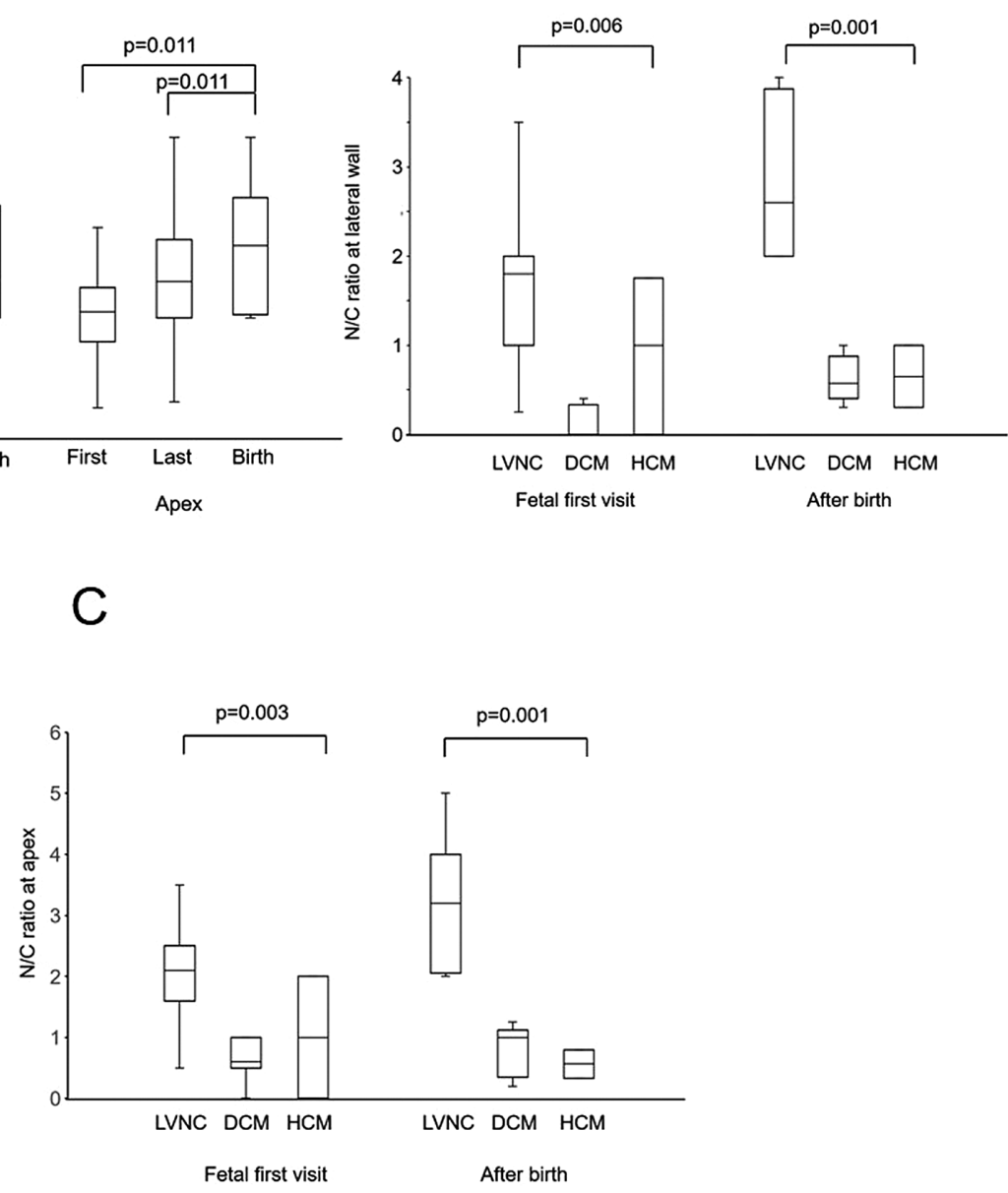

Figure 2. Serial echocardiographic changes in the N/C ratios through the prenatal to postnatal period. (A) Serial changes in the $\mathrm{N} / \mathrm{C}$ ratio at first prenatal visit (median 30 weeks of gestation (18-39weeks)), at last prenatal visit (median 36 weeks of gestation (27-39weeks)), and postnatal (birth) for patients with LVNC diagnosed after birth in the lateral wall (lefthand side) and apex (righthand side). (B) Serial changes in the N/C ratio at the lateral wall from the fetal first visit (lefthand side) to after birth (righthand side) among the 3 groups of cardiomyopathies. (C) Serial changes in N/C ratio at the apex from the fetal first visit (lefthand side) to after birth (righthand side) among the 3 groups of cardiomyopathies. DCM, dilated cardiomyopathy; HCM, hypertrophic cardiomyopathy; LVNC, left ventricular noncompaction; N/C, noncompacted-to-compacted.

months). The median gestational age at diagnosis was 30 weeks (18-39 weeks). Fetal cardiac screening was the most effective examination for detecting and diagnosing $\mathrm{CM}$ in the fetuses $(57.8 \%)$. Fetuses with LVNC had a significantly higher positive family history of CM than those with DCM and $\mathrm{HCM}(\mathrm{P}=0.020)$. Fetal complications, such as positive nuchal translucency and intrauterine growth restriction, were observed in 16 fetuses $(42.1 \%)$. The incidence of developing congenital heart disease (CHD) was significantly higher in fetuses with $\operatorname{LVNC}(9,56.3 \%)$ than in fetuses with DCM and HCM $(\mathrm{P}=0.024)$.

\section{Clinical Features After Birth}

Almost all infants were born at term (full-term birth); however, half were delivered by cesarean section (Table 2). Findings of extra cardiac involvement were most frequently observed in patients with HCM (42.8\%), whereas patients with LVNC had the highest incidence of arrhythmias $(26.6 \%)$. A total of 24 patients $(92.3 \%)$ with CM received treatment, including angiotensin-converting enzyme inhibitors, angiotensin-receptor blockers, $\beta$-blockers, catecholamines, phosphodiesterase III inhibitors, diuretics, antiplatelets, anticoagulants, ventilator support, and pacemaker implantation. No significant differences were observed between patients with CM for each type of treatment. However, compared with the patients with DCM and $\mathrm{HCM}$, patients with LVNC required multiple treatments.

\section{Echocardiographic Findings}

The echocardiographic characteristics at first visit are shown in Table 1. Mitral regurgitation (MR) of any grade was frequently observed in the fetuses with DCM compared with those with LVNC and HCM $(\mathrm{P}=0.0001)$. Echocardiographic findings after birth are given in Table 2. 


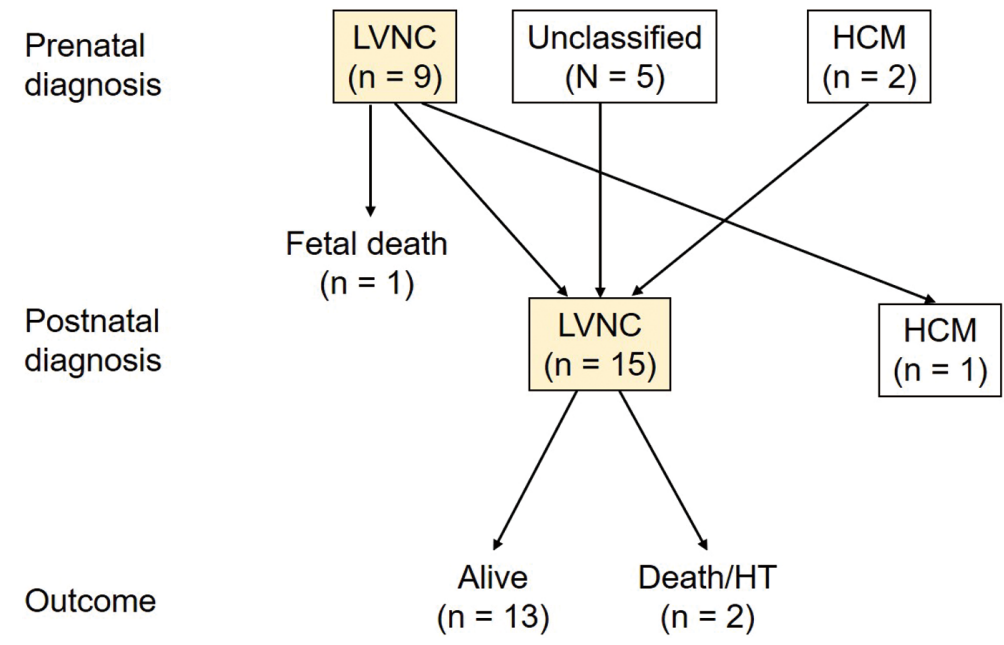

Figure 3. Flow chart of diagnosis of patients with LVNC before and after birth with their outcomes, showing discrepancies between prenatal and postnatal diagnosis and prognosis. HCM, hypertrophic cardiomyopathy; LVNC, left ventricular noncompaction.

Table 3. Univariate and Multivariate Cox Proportional-Hazards Regression Analysis for the Independent Predictors of HT or Death

\begin{tabular}{|c|c|c|c|c|c|c|}
\hline & \multicolumn{3}{|c|}{ Univariate analysis } & \multicolumn{3}{|c|}{ Multivariate analysis } \\
\hline & Odds ratio & $95 \% \mathrm{Cl}$ & $P$ value & Odds ratio & $95 \% \mathrm{Cl}$ & $P$ value \\
\hline Sex (male) & 12.0 & $1.30-110.52$ & 0.0072 & & & \\
\hline DCM diagnosis at birth & 16.5 & $3.09-88.03$ & 0.0002 & 18.7 & $1.40-248.39$ & 0.0265 \\
\hline CVPS $<7$ & 21.0 & $1.94-227.20$ & 0.0026 & 26.9 & $1.46-495.26$ & 0.0266 \\
\hline Prenatal MR & 7.5 & $1.60-34.95$ & 0.0065 & & & \\
\hline Postnatal MR (>moderate) & 18.9 & $2.54-141.92$ & 0.0015 & & & \\
\hline Pulmonary congestion at birth & 7.0 & $1.08-45.15$ & 0.0283 & & & \\
\hline
\end{tabular}

$\mathrm{Cl}$, confidence interval. Other abbreviations as in Tables 1,2.

Reduced LVFS was noted in patients with DCM $(13.1 \pm 8.9 \%, \mathrm{P}=0.027)$. Moderate and severe $\mathrm{MR}$ was more frequently detected in patients with DCM than in patients with LVNC and HCM ( $\mathrm{P}=0.005)$. The IVS z-score was significantly higher in patients with $\mathrm{HCM}$ than in patients with LVNC and DCM $(\mathrm{P}=0.012)$.

\section{Serial Echocardiographic Variables}

Prominent trabeculations were mostly observed in the lateral LV, posterior LV, and apex of the LV in patients with LVNC compared with those with DCM and HCM between the fetal and neonatal periods (Figure 2, Supplementary Figure 1). The N/C ratio was significantly higher in patients with LVNC than in patients with DCM and HCM at first visit (lateral $L V: 1.6 \pm 0.1,0.4 \pm 0.2$, and $0.5 \pm 0.4, \mathrm{P}=0.006$; LV apex: $2.0 \pm 0.1,0.6 \pm 0.2$, and $1.0 \pm 0.4, \mathrm{P}=0.003$, respectively). Of note, the $\mathrm{N} / \mathrm{C}$ ratio at the apex and lateral wall increased time-dependently from the fetal period to birth. After birth, the N/C ratio was significantly higher in patients with LVNC than in patients with DCM and HCM (lateral $\mathrm{LV}: 2.8 \pm 0.2,0.6 \pm 0.3$, and $0.6 \pm 0.5, \mathrm{P}=0.001$; LV apex: $3.2 \pm 0.2,0.7 \pm 0.4$, and $0.5 \pm 0.6, \mathrm{P}=0.001$, respectively). Thus, the N/C ratios after birth in the LVNC group were higher than 2.0 for both the lateral LV and LV apex, whereas the $\mathrm{N} / \mathrm{C}$ ratios in LVNC during the fetal period were slightly lower than 2.0.

\section{Concordance Between Prenatal and Postnatal Diagnoses}

We reviewed the prenatal diagnoses retrospectively and analyzed the concordance between the prenatal and postnatal diagnoses. The concordance rate for $\mathrm{CM}$ was $60.5 \%$; LVNC 56.3\%, DCM 73.3\%; HCM 42.9\% (Table 1, Figure 3, Supplementary Figure 2). Of 16 patients with LVNC, 9 $(56.3 \%)$ were diagnosed at the first visit; there was 1 intrauterine death and 2 other patients died, which was a higher mortality rate than in patients with HCM and a lower concordance rate than in patients with DCM.

\section{Prognosis of CM and Risk Factors for Death and LVNC}

During the period of the study, 16 patients died or underwent HT: 4 with LVNC, 11 DCM, 1 HCM (Table 1). Of these patients, 3 died during the fetal period: 1 with LVNC and 2 with DCM. No significant differences were observed in terms of other clinical parameters among the fetuses with CM. The multivariate proportional-hazards model, including fetal echocardiographic parameters, showed that final DCM diagnosis at birth and CVPS score $<7$ were independent risk factors for death or HT in all patients with CM (odds ratio (OR): 18.7, 95\% confidence interval (CI): 1.4-248.39, P=0.026; OR: 26.9, 95\% CI: 1.46-495.26, $\mathrm{P}=0.026$, respectively) (Table 3, Figure 4). In addition, CM patients with MR at the final visit had significantly higher mortality rates than those without MR during the prenatal period $(\mathrm{P}=0.0185)$ (Figure 4).

The multivariate proportional hazards model showed that $\mathrm{N} / \mathrm{C}$ ratio $\geq 1.6$ at the apex at the first visit in the fetus was a significant predictor of LVNC (OR: 47.8, 95\% CI: 2.39-956.39, $\mathrm{P}=0.0113$ ) (Table 4). The ROC curve was 


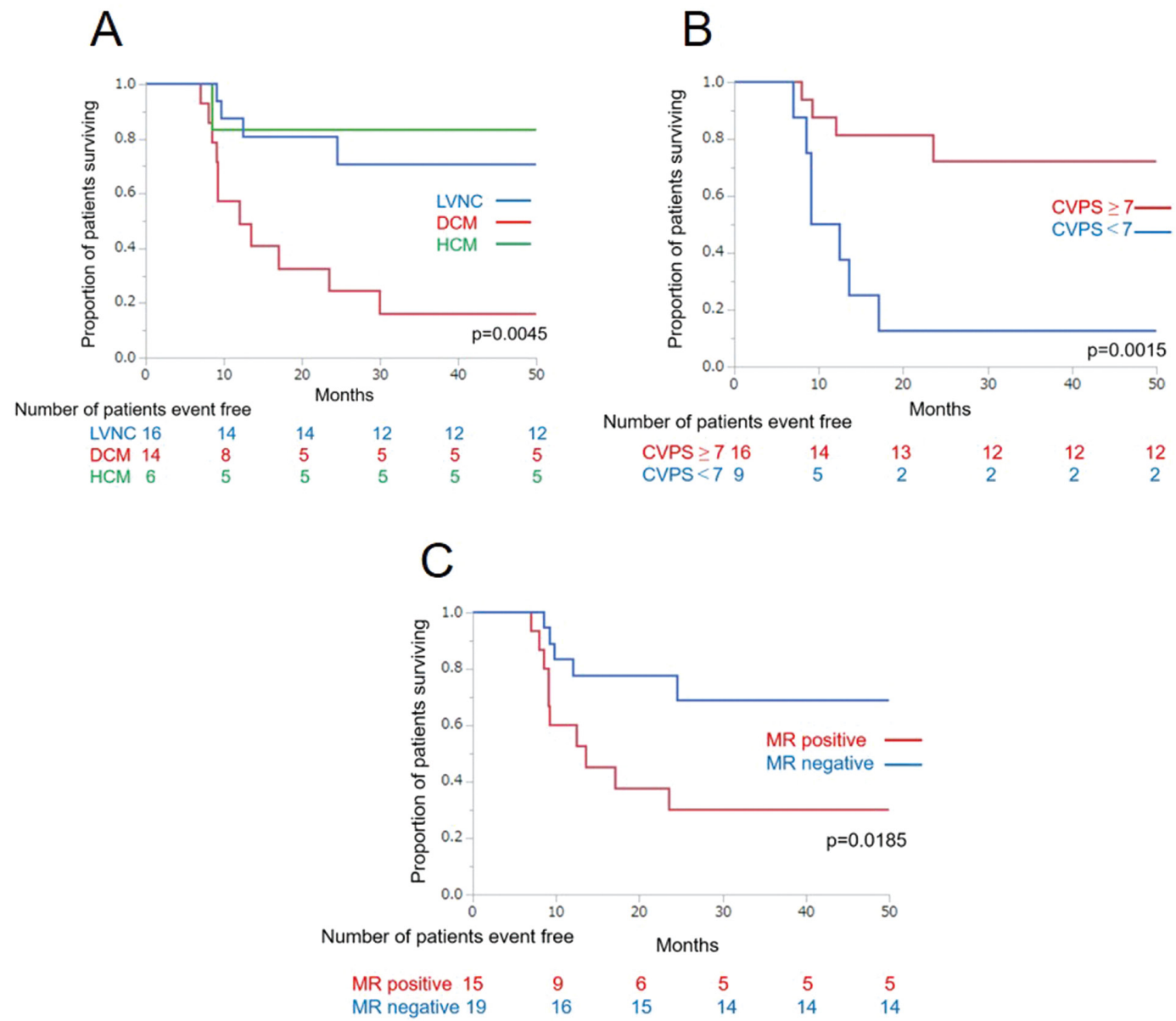

Figure 4. Event-free survival in patients with cardiomyopathies. Event-free survival to endpoints of death or HT in patients with cardiomyopathies according to type (A), CVPS at first prenatal visit (B), and MR at last prenatal visit (C). CVPS, cardiovascular profile score; DCM, dilated cardiomyopathy; HCM, hypertrophic cardiomyopathy; LVNC, left ventricular noncompaction; MR, mitral regurgitation.

Table 4. Univariate and Multivariate Cox Proportional-Hazards Regression Analysis for the Independent Predictors of Fetal LVNC

\begin{tabular}{|c|c|c|c|c|c|c|}
\hline & \multicolumn{3}{|c|}{ Univariate analysis } & \multicolumn{3}{|c|}{ Multivariate analysis } \\
\hline & Odds ratio & $95 \% \mathrm{Cl}$ & $P$ value & Odds ratio & $95 \% \mathrm{Cl}$ & $P$ value \\
\hline Family history of $\mathrm{CM}$ & 10.0 & $1.73-57.7$ & 0.0100 & & & \\
\hline Congenital heart disease & 8.14 & $1.69-39.05$ & 0.0088 & & & \\
\hline $\mathrm{N} / \mathrm{C}$ ratio $\geq 1.7$ at the lateral wall & 12.8 & $1.31-125.77$ & 0.0282 & & & \\
\hline $\mathrm{N} / \mathrm{C}$ ratio $\geq 1.6$ at the apex & 69.9 & $5.55-882.19$ & 0.0010 & 47.8 & 2.39-956.39 & 0.0113 \\
\hline
\end{tabular}

$\mathrm{N} / \mathrm{C}$, noncompacted-to-compacted. Other abbreviations as in Tables 1,3.

screened and the area under the curve of the $\mathrm{N} / \mathrm{C}$ ratio at the apex was 0.892 . The sensitivity and specificity were $87.5 \%$ and $90.9 \%$, respectively for the diagnosis of fetal LVNC (Supplementary Figure 3).

\section{Discussion}

In this study, we demonstrate the etiology of fetal CM from a large cohort in Japan. Moreover, this is the first longitudinal study of the clinical and echocardiographic features during the perinatal period in patients with
LVNC. Our study shows that reaching a definitive diagnosis during the fetal period is challenging, and highlights the need for new diagnostic criteria for LVNC because the prognosis varies according to the fetal onset of CM types.

According to the annual survey of the Japanese Society of Pediatric Cardiology and Cardiac Surgery (2005-2014), the occurrence of LVNC, DCM, and HCM in the whole pediatric populations was $28.7,43.1$, and 35.8 persons per year, respectively. ${ }^{11}$ Therefore, the occurrence of LVNC, DCM, and HCM in patients under 1 year of age was 12.6, 19.5 , and 10.0 persons per year, respectively. In this study, 
over 7 years, we collected the data of 38 patients from 20 hospitals out of 131 relevant hospitals in Japan. Thus, taking to account that the collection rate was $15.3 \%$, the estimated occurrence of LVNC, DCM, and HCM in fetuses in this study was $14.9,14.0$, and 6.5 fetuses per year, respectively. These data are compatible with data reported in the annual Japanese survey and showed that the populations of fetal onset of LVNC, DCM, and HCM with respect to the whole pediatric $\mathrm{CM}$ cohort were $51.9 \%, 32.4 \%$, and $18.1 \%$, respectively. The annual number of births was approximately 1,000,000 in Japan (2014), indicating that the incidence of babies with LVNC, DCM, and HCM was 1.49, 1.40 , and 0.65 per 100,000 babies, respectively.

Previous studies adopted the diagnostic criteria of LVNC in fetal patients that are used for children and adult patients with LVNC. ${ }^{7}$ However, our results showed that when these criteria are applied, there can be failure to diagnose some patients with LVNC in utero because the prominent trabeculations gradually appear in the later stage of pregnancy. It has been reported that CM may develop later in pregnancy even if earlier fetal echocardiography shows normal ventricular size and function. ${ }^{\mathbf{1 2}}$ Besides, the N/C ratio of the myocardium in the normal fetus is much higher than that in children and adults, ${ }^{\mathbf{1 3}}$ indicating that compaction of the fetal LV myocardium has not completely developed even in the late gestational stage and that compaction of the fetal LV develops gradually. ${ }^{14}$ Thus, our results showed that it is difficult to detect LVNC early in utero and highlighted the need for specific diagnostic criteria of fetal LVNC.

At 5-8 weeks of gestation, the ventricular myocardium gradually compacts. The densification of the fetal LV myocardium occurs gradually from the base to the apex and from the anterior to the lateral, posterior, and inferior walls. ${ }^{15}$ By the 12 th week of embryonic development, the compaction process is completed. ${ }^{\mathbf{1 6}}$ Failed compaction of embryonic trabeculations might lead to LVNC, whereby an excessive number of trabeculations linger in the ventricular lumen. ${ }^{17}$ However, our results did not support the hypothesis that arrest of the compaction of the LV wall leads to LVNC, because there were greater thickness of trabeculations in the late gestational stage than in the midgestational stage. A recent study reported that noncompaction results from abnormal growth of the compact wall. Alternatively, another study suggested that the excessive trabeculations in noncompaction do not have an embryonic identity and failed compaction probably does not result in noncompaction. ${ }^{18}$ Pressure overload of the $\mathrm{LV}$ is another possible mechanism. Cardiac output is reported to be $210 \mathrm{~mL} / \mathrm{min}$ and $1,900 \mathrm{~mL} / \mathrm{min} 20$ to 38 week gestation, respectively. ${ }^{19}$ From 30 - to 38 -week gestation, cardiac output increases two-fold. In adult patients, acquired LVNC occurs in athletes and patients with sickle cell anemia, pregnancy, myopathies, and chronic renal failure. ${ }^{20,21}$ During LV pressure overload, ${ }^{22}$ a change in LV morphology and a decrease in LV mass may occur, resulting in the manifestation of prominent trabeculations in the LV. As well as these previous observations, additional stress to the myocardium may trigger a deterioration of systolic function, resulting in prominent trabeculations in the LV during the fetal period, because LVNC is frequently associated with systolic dysfunction. All in all, our longitudinal observations from echocardiography in the fetus suggest a new hypothesis that physiological mechanical stress to an impaired LV myocardium, even during the fetal period, plays a role in the development of LVNC rather than the current hypothesis of developmental escape for maturity.

Our results revealed that high mortality rates and severe symptoms are associated with fetal LVNC. Fetuses with LVNC usually have enlarged and poorly functioning ventricles. ${ }^{7,23}$ A few studies only reported the successful outcomes of diagnosing LVNC in utero. A recent review of 106 published cases showed that only 46 patients with prenatally diagnosed LVNC were alive after birth, ${ }^{23}$ and the remaining 60 patients died due to termination of pregnancy or intrauterine death or during the first few weeks of postnatal life. Our results showed that CHD involvement was noted in 9 patients $(56.3 \%)$. LVNC is often associated with various $\mathrm{CHDs}$, many of which have serious anatomic and physiologic consequences leading to eventual cardiac failure and neonatal death, so even in isolation it a significant predictor of severe cardiac failure. ${ }^{7,24}$ Therefore, poor cardiac function during the fetal period and the complications of CHD may strongly affect the prognosis of fetal onset of LVNC.

\section{Study Limitations}

This was a retrospective study and most of the patients were referrals from outside facilities; therefore, there was no long-term follow-up of the patients. Moreover, due to the retrospective nature of the study, we only selected patients with LVNC diagnosed in utero. The present study was conducted over approximately 7 years, and, during this time, therapies have been developed that may have altered patient outcomes.

\section{Conclusions}

We demonstrated the etiology of fetal CM based on a nationwide survey conducted in Japan. Our results revealed that diagnosing LVNC in fetal patients is challenging despite the greater caution of LVNC with poor prognosis. Early detection of LVNC will result in early intervention and treatment to delay the progression of heart failure and improve the quality of life. To better understand and manage fetal CM, novel diagnostic criteria of LVNC in a fetus should be established. Further large-scale cohort studies with genetic testing and high-resolution modalities, such as magnetic resonance imaging, are warranted.

\section{Acknowledgments}

The authors acknowledge the expert technical assistance of Hitoshi Moriuchi, Haruna Hirai and Eriko Masuda for.

\section{Funding}

This study was supported financially by JSPS KAKENHI Grant number $16 \mathrm{~K} 10063$.

\section{Disclosure}

The authors declare no conflicts of interest.

\section{IRB Information}

The study protocols were approved by the Research Ethics Committee of the University of Toyama (approval no. R2016020).

\section{References}

1. Chin TK, Perloff JK, Williams RG, Jue K, Mohrmann R. Isolated noncompaction of left ventricular myocardium: A study of eight cases. Circulation 1990; 82: 507-513.

2. Pignatelli RH, McMahon CJ, Dreyer WJ, Denfield SW, Price J, Belmont JW, et al. Clinical characterization of left ventricular noncompaction in children: A relatively common form of cardio- 
myopathy. Circulation 2003; 108: 2672-2678.

3. Ichida F, Hamamichi Y, Miyawaki T, Ono Y, Kamiya T, Akagi $\mathrm{T}$, et al. Clinical features of isolated noncompaction of the ventricular myocardium: Long-term clinical course, hemodynamic properties, and genetic background. J Am Coll Cardiol 1999; 34: $233-240$.

4. Ichida F, Tsubata S, Bowles KR, Haneda N, Uese K, Miyawaki $\mathrm{T}$, et al. Novel gene mutations in patients with left ventricular noncompaction or Barth syndrome. Circulation 2001; 103: 1256 1263.

5. Aras D, Tufekcioglu O, Ergun K, Ozeke O, Yildiz A, Topaloglu $\mathrm{S}$, et al. Clinical features of isolated ventricular noncompaction in adults: Long-term clinical course, echocardiographic properties, and predictors of left ventricular failure. J Card Fail 2006; 12: $726-733$.

6. Stollberger C, Wegner C, Benatar A, Chin TK, Dangel J, Majoor-Krakauer D, et al. Postnatal outcome of fetal left ventricular hypertrabeculation/noncompaction. Pediatr Cardiol 2016; 37: $919-924$

7. Arunamata A, Punn R, Cuneo B, Bharati S, Silverman NH. Echocardiographic diagnosis and prognosis of fetal left ventricular noncompaction. J Am Soc Echocardiogr 2012; 25: 112-120.

8. Jenni R, Rojas J, Oechslin E. Isolated noncompaction of the myocardium. N Engl J Med 1999; 340: 966-967.

9. Pettersen MD, Du W, Skeens ME, Humes RA. Regression equations for calculation of $\mathrm{z}$ scores of cardiac structures in a large cohort of healthy infants, children, and adolescents: An echocardiographic study. J Am Soc Echocardiogr 2008; 21: 922-934.

10. Huhta JC. Fetal congestive heart failure. Semin Fetal Neonatal Med 2005; 10: 542-552.

11. Hirono K, Miyao N, Kobayashi T, Moriichi A, Takarada S, Okabe M, et al. Clinical features of noncompaction cardiomyopathy: A questionnaire-based survey in Japan. Ped Cardiol Card Surg 2019; 35: 172-178.

12. Yinon Y, Yagel S, Hegesh J, Weisz B, Mazaki-Tovi S, Lipitz S, et al. Fetal cardiomyopathy: In utero evaluation and clinical significance. Prenat Diagn 2007; 27: 23-28.

13. Jensen B, Agger P, de Boer BA, Oostra RJ, Pedersen M, van der Wal AC, et al. The hypertrabeculated (noncompacted) left ventricle is different from the ventricle of embryos and ectothermic vertebrates. Biochim Biophys Acta 2016; 1863: 1696-1706.

14. Bai Y, Liu B, Wei Y. Detection of thickness and ratio of left ventricular myocardial noncompaction and compaction layers in normal children by two-dimensional echocardiography. $J$ Ultrasound Clin Med 2010; 12: 818-820.

15. Sun L, Zhao E, Wei Y, Kang C, Liu B. Thickness and ratio of noncompacted and compacted layers of the left ventricular myocardium evaluated in 56 normal fetuses by two-dimensional echocardiography. Biomed Res Int 2019; 2019: 3726846.

16. Sedmera D, Pexieder T, Vuillemin M, Thompson RP, Anderson RH. Developmental patterning of the myocardium. Anat Rec 2000; 258: 319-337.

17. Towbin JA, Lorts A, Jefferies JL. Left ventricular non-compaction cardiomyopathy. Lancet 2015; 386: 813-825.

18. Jensen B, van der Wal AC, Moorman AFM, Christoffels VM. Excessive trabeculations in noncompaction do not have the embryonic identity. Int J Cardiol 2017; 227: 325-330.

19. Rasanen J, Wood DC, Weiner S, Ludomirski A, Huhta JC. Role of the pulmonary circulation in the distribution of human fetal cardiac output during the second half of pregnancy. Circulation 1996; 94: $1068-1073$.

20. Gati S, Papadakis M, Papamichael ND, Zaidi A, Sheikh N, Reed $\mathrm{M}$, et al. Reversible de novo left ventricular trabeculations in pregnant women: Implications for the diagnosis of left ventricular noncompaction in low-risk populations. Circulation 2014; 130: $475-483$.

21. Gati S, Papadakis M, Van Niekerk N, Reed M, Yeghen T, Sharma S. Increased left ventricular trabeculation in individuals with sickle cell anaemia: Physiology or pathology? Int J Cardiol 2013; 168: $1658-1660$.

22. Struijk PC, Mathews VJ, Loupas T, Stewart PA, Steegers EAP, Wladimiroff JW. Blood pressure estimation in the human fetal descending aorta. Ultrasound Obstet Gynecol 2008; 32: 673-681.

23. Stollberger C, Wegner C, Finsterer J. Fetal ventricular hypertrabeculation/noncompaction: Clinical presentation, genetics, associated cardiac and extracardiac abnormalities and outcome. Pediatr Cardiol 2015; 36: 1319-1326.

24. Ursell PC. Noncompaction in the fetus and neonate: An autopsy study. Am J Med Genet C Semin Med Genet 2013; 163C: 169177.

\section{Supplementary Files}

Please find supplementary file(s);

http://dx.doi.org/10.1253/circj.CJ-20-1148 